\title{
Editorial: Rehabilitation Bioengineering
}

We are pleased to present a special issue on the topic of rehabilitation bioengineering. Rehabilitation bioengineering is the application of science and technology to ameliorate the disabilities of individuals. Since our society is becoming older and costs for healthcare are continuously increasing, the development of reliable and cost-efficient medical and rehabilitation systems poses a great challenge, internationally. Rehabilitation bioengineering plays a major role in providing solutions for individuals affected by sensory loss, motor impairments or communication difficulties. It also opens new frontiers in our understanding of medical disorders and their long-term effects and impact on the quality of life. Past and current advances in musculoskeletal biomechanics, robotics, computational modeling, tissue mechanics, communication, humanmachine interfaces and more, enable patients undergoing rehabilitation to independently return to their daily life activities.

Rehabilitation bioengineering can be viewed as an interface for a wide variety of technical, physiological and other concerns. The rehabilitation engineer may be confronted with a hemiplegic stroke survivor, whose motor impairments are expressed by foot drop, causing gait asymmetries and frequent stumbles or falls. Also, restricted arm and hand movements on the paretic side may prevent the patient from participating in daily life activities and conducting an independent lifestyle. Furthermore, language or communication skills may be impaired. Several state-of-the art technological solutions have been developed to cope with some specific impairments, but typically a holistic approach to rehabilitation technology in such cases will be lacking. Moreover, finding the optimal integrative solution, specific for each individual, proves to be a challenge. For example, a rehabilitation professional is often confronted with a dilemma, especially when numerous solutions can be considered for the same patient: functional electrical stimulation, orthotics, robotics, or a combination of the above. The same dilemma applies to upper extremity rehabilitation and communication, but different solutions may result in different outcomes for different individuals. The selection of the optimal rehabilitation plan depends on our evaluation tools, their sensitivity, reliability and validity, as well as on the available treatment modalities.

This example is only a glimpse into the many challenges and prospects that await the original and novel solutions supplied by the biomedical engineers and others involved in the rehabilitation process of individuals with disabilities. Hence, this special issue focuses on the new and exciting methods and applications related to rehabilitation bioengineering, designed for patient evaluation, diagnosis and treatment at the hospital, community and home settings. The topics discussed in this issue branch out to various disciplines that were recently transformed by biomedical engineers, such as wound electrotherapy, orthotics, muscle regeneration, computational biomechanics, tissue biomechanics, rehabilitation of vision and more. Advances in the field of rehabilitation bioengineering are described in this issue by eminent groups in institutes across Europe, the US, Israel, Australia and Asia which depicts the international research frontiers and their diversity in this exciting, highly important arena.

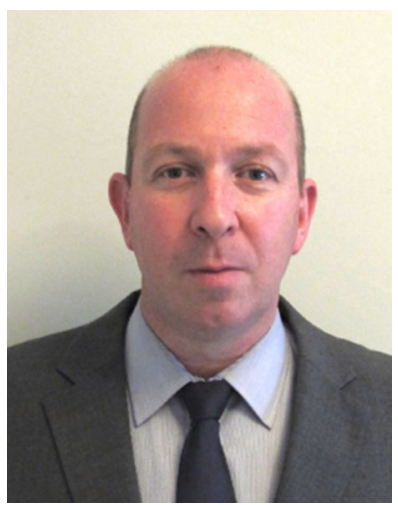

AlON Wolf

Faculty of Mechanical

Engineering

Technion - Israel

Institute of Technology,

Hafia, Israel

Electronic mail:

alonw@technion.ac.il 
Wolf et al.

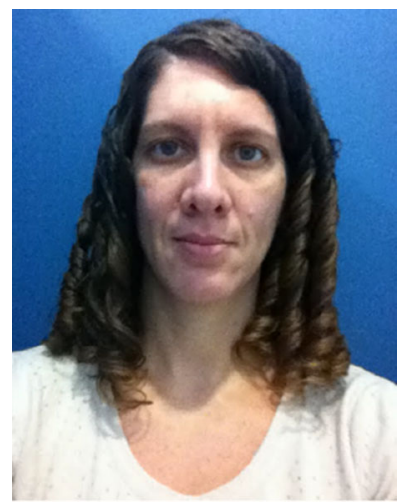

Sigal Portnoy

Department of

Occupational Therapy,

Tel Aviv University,

Tel Aviv, Israel

Electronic mail:

portnoys@post.tau.ac.il

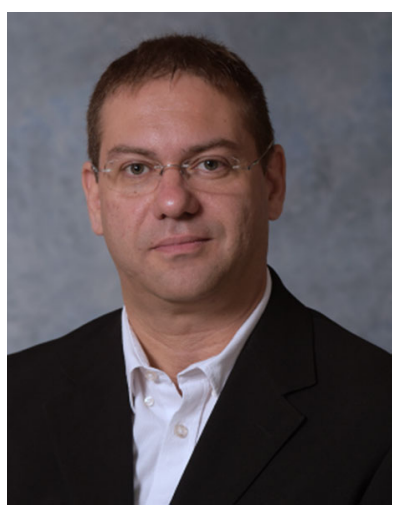

\section{Amit Gefen}

Department of

Biomedical Engineering, Faculty of Engineering,

Tel Aviv University,

Tel Aviv, Israel

Electronic mail:

gefen@eng.tau.ac.il 